\title{
Reduced Field-of-View Diffusion Tensor Imaging of the Optic Nerve in Retinitis Pigmentosa at $3 T$
}

\author{
(D) Y. Zhang, (D)X. Guo, (D) M. Wang, (D). Wang, (D). Tian, DD. Zheng, and DD. Shi
}

\begin{abstract}
BACKGROUND AND PURPOSE: Diffusion tensor imaging may reflect pathology of the optic nerve; however, the ability of DTI to evaluate alterations of the optic nerve in retinitis pigmentosa has not yet been assessed, to our knowledge. The aim of this study was to investigate the diagnostic potential of reduced FOV-DTI in optic neuropathy of retinitis pigmentosa at 3T.
\end{abstract}

MATERIALS AND METHODS: Thirty-eight patients and thirty-five healthy controls were enrolled in this study. Measures of visual field and visual acuity of both eyes in all subjects were performed. A reduced FOV-DTI sequence was used to derive fractional anisotropy, apparent diffusion coefficient, principal eigenvalue, and orthogonal eigenvalue of the individual optic nerves. Mean fractional anisotropy, ADC, and eigenvalue maps were obtained for quantitative analysis. Further analyses were performed to determine the correlation of fractional anisotropy, ADC, principal eigenvalue, and orthogonal eigenvalue with optic nerves in patients with mean deviation of the visual field and visual acuity, respectively.

RESULTS: The optic nerves of patients with retinitis pigmentosa compared with control subjects showed significantly higher ADC, principal eigenvalue, and orthogonal eigenvalue and significantly lower fractional anisotropy $(P<.01)$. For patients with retinitis pigmentosa, the mean deviation of the visual field of the optic nerve was significantly correlated with mean fractional anisotropy $(r=0.364, P=$ $.001)$ and orthogonal eigenvalue $(r=-0.254, P=.029)$, but it was not correlated with mean ADC $(P=.154)$ and principal eigenvalue $(P=$ .337). Moreover, no correlation between any DTI parameter and visual acuity in patients with retinitis pigmentosa was observed $(P>.05)$.

CONCLUSIONS: Reduced FOV-DTI measurement of the optic nerve may serve as a biomarker of axonal and myelin damage in optic neuropathy for patients with retinitis pigmentosa.

ABBREVIATIONS: FA = fractional anisotropy; $\lambda \perp=$ orthogonal eigenvalue; $\lambda / /=$ principal eigenvalue; $M D V F=$ mean deviation of the visual field; $O N=$ optic nerve; $\mathrm{rFOV}=$ reduced FOV; $\mathrm{RP}=$ retinitis pigmentosa; $\mathrm{VA}=$ visual acuity; $\mathrm{VF}=$ visual field

$\mathbf{R}$ etinitis pigmentosa (RP) is a common refractory visual disease and accounts for a large proportion of hereditary visual impairment. It is characterized by the progressive death of rod and cone photoreceptors, which leads to corresponding visual field (VF) defects. ${ }^{1}$ As a heterogeneous group of inherited retinal degenerative diseases, ${ }^{2} \mathrm{RP}$ displays extreme genetic heterogeneity. More than 80 disease genes have been identified so far, 58 of which

Received September 7, 2015; accepted after revision February 6, 2016 From the Departments of Radiology (Y.Z., M.W., L.W., Q.T., D.S.) and Ophthalmology (X.G.), Zhengzhou University People's Hospital, Henan Provincial People's Hospital, Zhengzhou, China; and GE Healthcare (D.Z.), Beijing, China.

This study was supported by the National Natural Science Foundation of China Grant (No. 81271534).

Please address correspondence to Dapeng Shi, MD, Zhengzhou University People's Hospital, Henan Provincial People's Hospital, Department of Radiology, \#7 Wei Wu Rd, Zhengzhou, Henan 450003, China; e-mail: cjr.shidapeng@vip.163.com

- Indicates open access to non-subscribers at www.ajnr.org

http://dx.doi.org/10.3174/ajnr.A4767 correspond to nonsyndromic RP. ${ }^{3}$ Clinical features of RP include night blindness, progressive loss of peripheral VFs, reduced or nondetectable electroretinogram amplitudes, and characteristic pigmentary degenerative changes of the retina.

Several studies observed that the retinal nerve fiber layer, formed by expansion of optic nerve (ON) fibers, is significantly thinner in patients with RP by using optical coherence tomography, ${ }^{4,5}$ which reflects the changes of the ON in RP. Furthermore, the occurrence of optic neuropathy in RP was confirmed by a postmortem study that showed that total axon counts of the ON were significantly decreased in patients with end-stage RP compared with healthy controls. ${ }^{6}$ However, in vivo diagnosis of optic neuropathy in patients with RP remains challenging because conventional MR imaging and ophthalmologic examinations often fail to detect ON disease.

Recently, DTI has emerged as a noninvasive imaging method with great potential to investigate the morphology and function of the ON in vivo. ${ }^{7,8}$ However, there has been no study of DTI in 


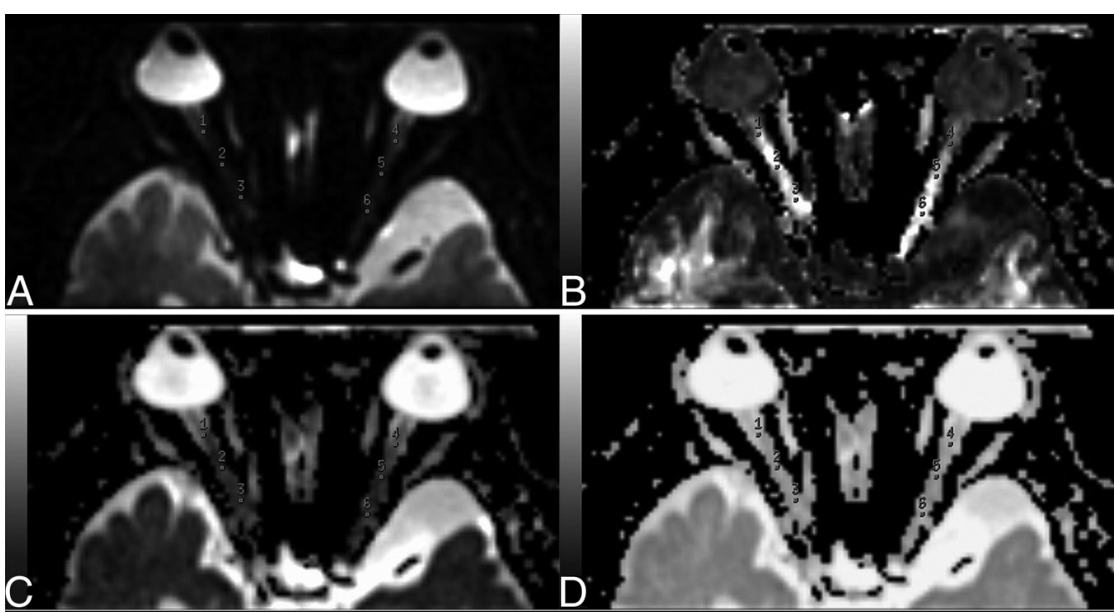

FIG 1. Non-diffusion-weighted (BO) image and FA, ADC, and eigenvector maps of DTI. Three ROIs are placed over each intraorbital nerve on the $b=0$ image $(A)$. ROls on the $b=0$-averaged image $(A)$ are then transferred onto the FA $(B), A D C(C)$, and eigenvector $(D)$ maps.

optic neuropathy of RP to date, especially reduced FOV-DTI (rFOV-DTI) at 3T, which has the potential advantages of improved signal-to-noise ratio and reduced susceptibility-related artifacts over conventional DTI. ${ }^{9,10}$ This study set out to investigate the potential clinical utility of rFOV-DTI in diagnosing optic neuropathy in RP at 3T. Specifically, we hypothesized that quantitative rFOV-DTI might reveal the injury to the ON of RP, which may be related to visual functions, including both VF and visual acuity (VA).

\section{MATERIALS AND METHODS \\ Subjects}

Thirty-eight patients (24 males, 14 females; mean age, 37 years; range, 6-62 years) with RP were recruited for this study from August 2014 to September 2015 from Zhengzhou University People's Hospital. No patient had any potentially contributory history of trauma, poisoning, or metabolic or autoimmune diseases. Each patient underwent a complete ophthalmologic examination. Diagnosis of RP was made according to the following criteria: 1) a history of night blindness, with gradual loss of VA during the day; 2) progressive VF defects, from peripheral to central, leading to typical tunnel vision; 3 ) typical fundus changes: optic disc pallor, attenuated retinal arterioles, and peripheral intraretinal pigment deposits in a bone-spicule configuration; and 4) abnormal findings on electroretinography: moderately reduced or almost nondetectable amplitudes of $\mathrm{a}$ - and b-waves and prolonged time intervals from stimuli to peak rod or cone isolated responses. ${ }^{4,5,11,12}$ Patients who had other ocular diseases, such as age-related macular degeneration, ischemic optic neuropathy, glaucoma, diabetic retinopathy, and optic neuritis were excluded. ${ }^{1,7,8}$

Thirty-five healthy volunteers (22 males, 13 females; mean age, 36 years; range, 9-60 years) were enrolled as a control group. All healthy volunteers completed formal ophthalmologic evaluation, including VA and VF. No healthy volunteers had any ophthalmologic or neurologic disorders. The bilateral corrected VA of healthy subjects was $\geq 0.8$.

This study was approved by the local ethics committee, and all subjects provided informed consent in writing in accordance with the Declaration of Helsinki.

\section{MR Imaging Protocol}

A $3 \mathrm{~T}$ whole-body scanner (Discovery MR 750; GE Healthcare, Milwaukee, Wisconsin) equipped with an 8-channel head coil was used, and subjects were instructed to close their eyes and remain still during the MR imaging study. Routine MR imaging of the brain and orbits was performed to exclude intracranial and intraorbital diseases. A 1-mm isotropic-resolution anatomic 3D T1weighted acquisition based on a magnetization-prepared rapid acquisition of gradient echo sequence was acquired. For the rFOV-DTI acquisition, a diffusion-weighted spin-echo single-shot echo-planar imaging with a 2D excitation pulse was used. Thirty noncollinear diffusion directions with $b=600 \mathrm{~s} / \mathrm{mm}^{2}$ and a $\mathrm{B} 0$ image were acquired with the following parameters: $\mathrm{TR}=$ $1800 \mathrm{~ms}, \mathrm{TE}=90 \mathrm{~ms}$, FOV $=16 \times 8 \mathrm{~cm}^{2}$, matrix $=96 \times 48$, section thickness $=2 \mathrm{~mm}$ with a $0-\mathrm{mm}$ section gap, NEX $=6$. The DTI scanning plane was oriented approximately parallel to the $\mathrm{ON}$ with the scanned volume from the infraorbital rim to the supraorbital rim. The acquisition time of rFOV-DTI was 5 minutes 37 seconds.

\section{MR Imaging Postprocessing}

Postprocessing of rFOV-DTI was performed by using FuncTool software (GE Healthcare) on an ADW4.5 workstation (GE Healthcare). Fractional anisotropy (FA), ADC, and eigenvector maps were calculated on a pixel-by-pixel basis. Each ON was manually segmented by 2 experienced neuroradiologists blinded to the clinical conditions of the patient, on the basis of the section of the B0 images where the intraorbital nerve could be clearly seen. To further reduce measurement errors, we manually drew 3 ROIs (each with an area of $2 \mathrm{~mm}^{2}$ ) at the level of intraorbital ON on the $\mathrm{B} 0$ image. ROIs were then transferred to the corrected FA, ADC, and eigenvector maps (Fig 1). For each ON, mean FA, ADC, principal eigenvalue $\left(\lambda / /=\lambda_{1}\right)$, and orthogonal eigenvalue $\left[\lambda \perp=\left(\lambda_{2}+\lambda_{3}\right) / 2\right]^{13}$ were calculated. The routine orbital MR images of patients with RP were reviewed by 2 experienced neuroradiologists.

\section{Ophthalmologic Examination}

The Humphrey Visual Field Analyzer (Carl Zeiss Meditec, Dublin, California) was used to assess the VF, and analysis was performed while pupils were in natural state. Subjects were instructed to keep their eyes open and focus their vision on the central target to the best of their ability. The threshold testing program 30-2 was selected to detect central $30^{\circ} \mathrm{VF}$ with sighting mark III and background 31.5asb. Results with fixation losses of $<20 \%$, false-negatives of $<33 \%$, and false-positives of $<15 \%$ were considered reliable. ${ }^{12}$ The test was performed twice, and the more reliable result was used for analysis, to reduce learning effects. The data were only included in the study if reliable data were obtained in repeat tests. The mean deviation of the visual field (MDVF) was obtained for quantitative analysis. The best corrected VA was measured 
with the international standard VA chart and was converted to a logarithm of minimum angle of resolution.

\section{Statistical Analysis}

Statistical analyses were performed by using SPSS 17.0 (IBM, Armonk, New York). For DTI measurement, differences between patients and controls and differences between right and left eyes were both evaluated by using an independent-samples $t$ test and paired $t$ test, respectively. Spearman rank correlations were calculated between DTI parameters (FA, ADC, $\lambda / /$, and $\lambda \perp$ ) and visual function (MDVF and VA) in patients with RP. $P$ values $<.05$ were considered statistically significant.

\section{RESULTS}

Epidemiologic and pathologic information of all 38 patients and 35 controls are shown in Table 1 . The results of orbital MR imaging are shown in Fig 2, and no morphologic and signal-intensity abnormality of the ONs in patients with RP was observed. All DTI measurements from patients and controls are summarized in Table 2. We made the following observations:

1) Mean FA from the right ONs in the patient group was lower than that from homolateral nerves in the control group $(P<$ .001 ), and the mean FA from the left ONs in the patient group was

lower than that from the homolateral nerves in the control group $(P<.001)$.

2) Mean $\mathrm{ADC}$ from the right $\mathrm{ONs}$ in the patient group was higher than that from the homolateral nerves in the control group $(P<.001)$, and mean ADC from the left ONs in the patient group was higher than that from the homolateral nerves in the control group $(P<.001)$.

3) Mean $\lambda / /$ from the right ONs in the patient group was higher than that from the homolateral nerves in the control group $(P=0.003)$, and mean $\lambda / /$ from the left ONs in the patient group was higher than that from the homolateral nerves in the control group $(P=.001)$.

4) Mean $\lambda \perp$ from the right ONs in the patient group was higher than that from the homolateral nerves in the control group $(P<.001)$, and mean $\lambda \perp$ from the left ONs in the patient group was higher than that from the homolateral nerves in the control group $(P<.001)$.

5) All DTI parameters (FA, ADC, $\lambda / /, \lambda \perp)$ between the right and left nerves in patients showed no significant differences $(P>$ $.05)$.

DTI measurements of both right and left nerves of the patients were compared with those of the controls (Fig 3). Significant correlations between MDVF and mean FA $(r=0.364, P=.001)$ and between MDVF and $\lambda \perp(r=-0.254$, $P=$.029) (Fig 4) were observed for patients with RP, but no correlation between MDVF and mean $\operatorname{ADC}(P=.154)$ or between MDVF and $\lambda / /(P=.337)$ was seen. Moreover, none of DTI parameters for ONs in RP were correlated with VA $(P>$ $.05)$.

\begin{tabular}{cccc}
$R$ & $-30.26 \pm 8.94(-37.00 \sim-4.68)$ & $-0.69 \pm 0.98(-2.00 \sim 1.23)$ & $.000^{c}$ \\
$L$ & $-29.41 \pm 9.73(-35.68 \sim-3.85)$ & $-0.54 \pm 1.15(-2.71 \sim 1.45)$ & $.000^{c}$ \\
VA (LogMAR) & & & \\
$R$ & $0.52 \pm 0.70(0 \sim 2)$ & $-0.067 \pm 0.08(-0.2 \sim 0.1)$ & $.000^{c}$ \\
$L$ & $0.52 \pm 0.68(0 \sim 2)$ & $-0.065 \pm 0.072(-0.2 \sim 0.1)$ & $.000^{c}$ \\
\hline
\end{tabular}

Note:-LogMAR indicates logarithm of minimal angle of resolution; $N_{R}$, number of right eyes; $N_{L}$, number of left eyes; $\mathrm{R}$, right eye; L, left eye; /, not available.

a Values are mean $\pm \mathrm{SD}$ (range).

${ }^{b}$ Values from patient group are median \pm interquartile range (range), and values from control group are mean $\pm \mathrm{SD}$ (range). c Significant difference is noted between patients and controls.
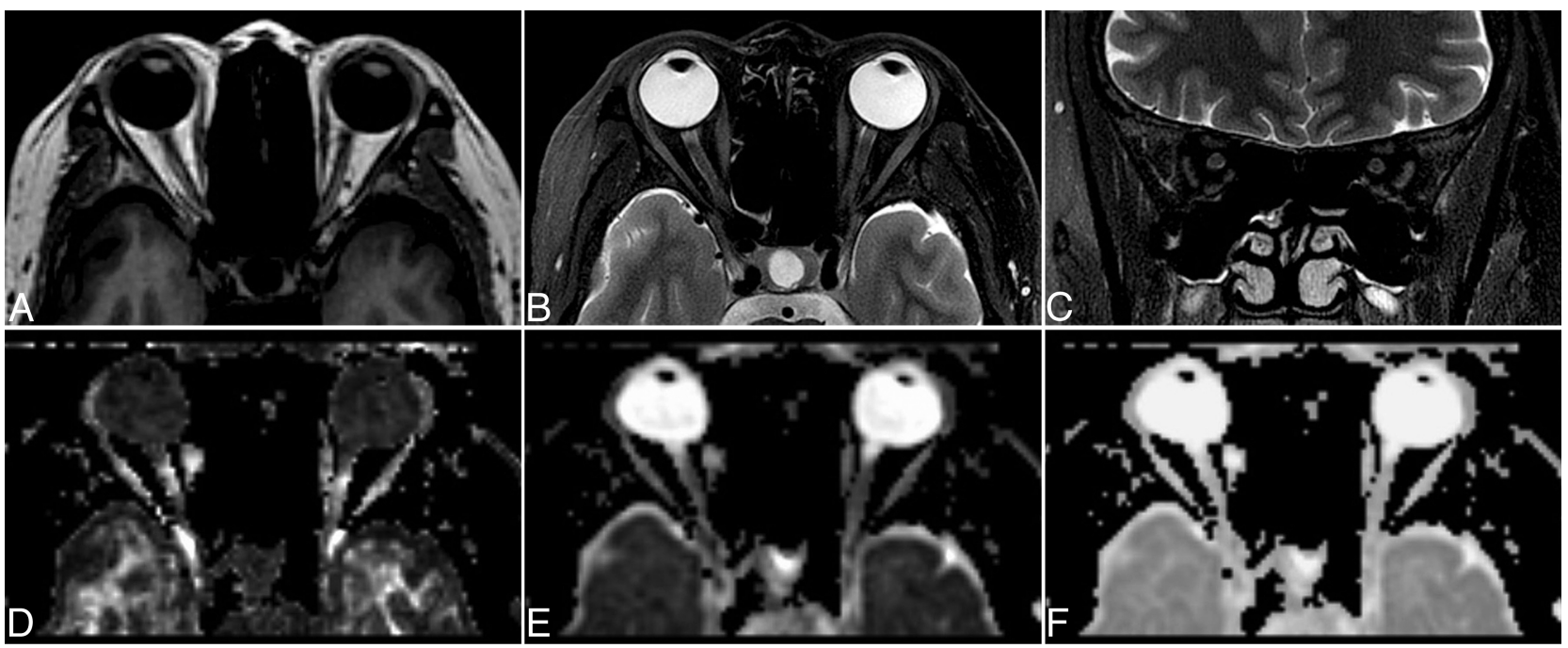

FIG 2. Routine orbital MR imaging of patients with RP together with DTI maps. Axial TIWI (A), axial fat-saturated T2WI (B), coronal fat-saturated T2WI $(C)$, and the corresponding FA (D), ADC (E), and eigenvector maps $(F)$ of DTI in a 48-year-old patient with RP. 
Table 2: DTI parameter data ${ }^{a}$ of patients with RP and controls

\begin{tabular}{lcccc}
\hline & Patients $(\boldsymbol{n}=38)$ & Controls $(\boldsymbol{n}=35)$ & $\boldsymbol{t}$ Value & $\boldsymbol{P}$ Value \\
\hline FA & & & & \\
$\quad \mathrm{R}$ & $0.442 \pm 0.077$ & $0.572 \pm 0.046$ & -8.840 & $.000^{\mathrm{b}}$ \\
$\mathrm{L}$ & $0.447 \pm 0.067$ & $0.581 \pm 0.044$ & -10.083 & $.000^{\mathrm{b}}$ \\
$\mathrm{ADC}\left(\times 10^{-3} \mathrm{~mm}^{2} \mathrm{~s}^{-1}\right)$ & & & \\
$\quad \mathrm{R}$ & $1.367 \pm 0.250$ & $1.099 \pm 0.166$ & 5.417 & $.000^{\mathrm{b}}$ \\
$\mathrm{L}$ & $1.343 \pm 0.218$ & $1.068 \pm 0.122$ & 6.667 & $.000^{\mathrm{b}}$ \\
$\lambda / /\left(\times 10^{-3} \mathrm{~mm}^{2} \mathrm{~s}^{-1}\right)$ & & & & \\
$\mathrm{R}$ & $5.548 \pm 0.288$ & $5.361 \pm 0.231$ & 3.030 & $.003^{\mathrm{b}}$ \\
$\mathrm{L}$ & $5.521 \pm 0.250$ & $5.333 \pm 0.191$ & 3.609 & $.001^{\mathrm{b}}$ \\
$\lambda \perp\left(\times 10^{-3} \mathrm{~mm}^{2} \mathrm{~s}^{-1}\right)$ & & & & \\
$\mathrm{R}$ & $4.526 \pm 0.249$ & $4.218 \pm 0.146$ & 6.511 & $.000^{\mathrm{b}}$ \\
$\mathrm{L}$ & $4.503 \pm 0.213$ & $4.187 \pm 0.106$ & 8.041 & $.000^{\mathrm{b}}$ \\
\hline
\end{tabular}

Note:- $R$ indicates right eye; $L$, left eye.

a Values are mean \pm SD.

${ }^{\mathrm{b}}$ Significant difference between patient nerves and control nerves as determined by using an independent-samples $t$ test.

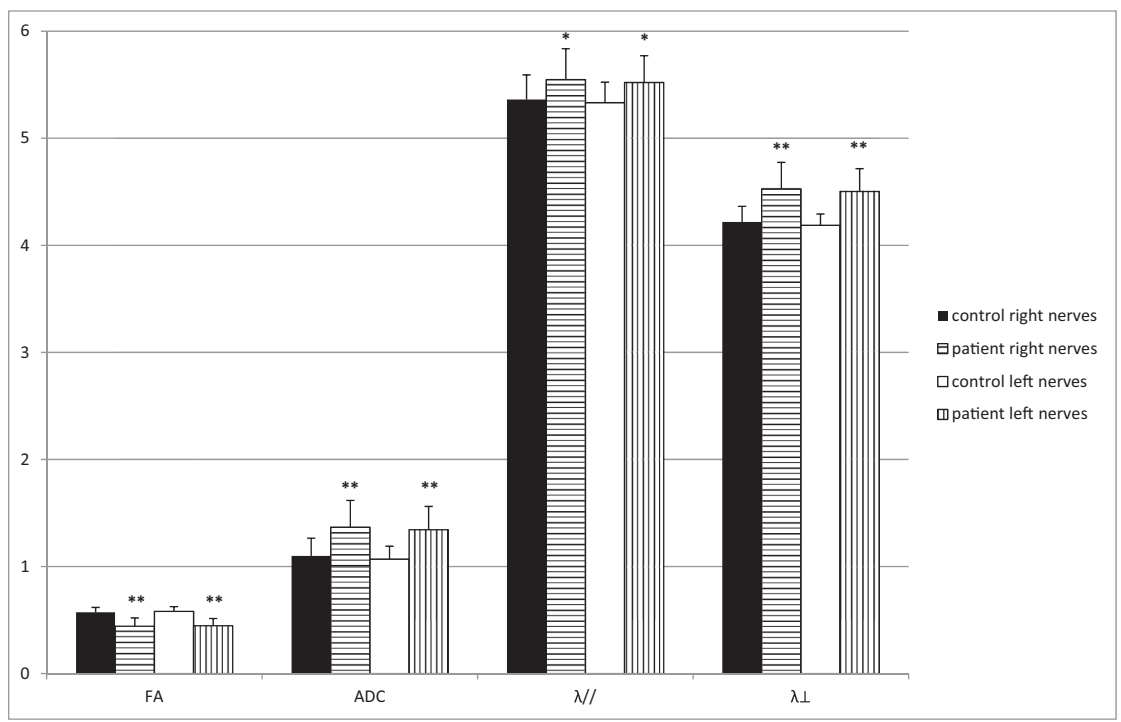

FIG 3. The DTI measures of both right and left ONs of patients are compared with corresponding normal ONs of controls. Bar graphs of mean FA, ADC, principal eigenvalue, and orthogonal eigenvalue averaged across the right and left optic nerves are shown in 38 patients with RP compared with the homolateral optic nerves of 35 controls. The error bars denote the SDs across subjects. The units of the ADC, $\lambda / /$, and $\lambda \perp$ measures are in $\times 10^{-3} \mathrm{~mm}^{2}$ per second ${ }^{-1}$. The asterisk indicates a significant difference compared with the homolateral nerves of controls $\left({ }^{\star} P<.01 ;{ }^{*} P<.001\right)$.

normality, whereas DTI measurements showed that mean ADC, $\lambda / /$, and $\lambda \perp$ for ONs in patients with RP were higher than those of controls, and mean FA was lower than that of controls. Hence, rFOV-DTI measurements convey potentially useful information in the diagnosis of optic neuropathy in RP. Furthermore, FA and $\lambda \perp$ respectively decreased and increased with the reduced VF.

In vivo DTI of the $\mathrm{ON}$ is challenging in practice due to its small dimension, which increases the vulnerability to motion and magnetic susceptibility artifacts. The effect of the hyperintense signal from surrounding orbital fat and CSF is also pronounced. ${ }^{7,8,14,15}$ In our study, rFOV-DTI was achieved with $90^{\circ}$ $2 \mathrm{D}$ echo-planar radiofrequency excitation followed by a refocusing $180^{\circ}$ pulse at $3 \mathrm{~T}$. This technique allows multisection imaging, and contiguous sections using this method can be obtained with no section gap as needed in zonal oblique multisection EPI. ${ }^{16}$ Also, the SNR does not depend on the number of sections because neighboring sections are not significantly excited by the $2 \mathrm{D}$ excitation pulse. Consequently, rFOV 2D excitation, compared with conventional excitation, is more advantageous in alleviating several problematic issues. ${ }^{9,10,16,17}$ When we compared the measurements of heathy subjects with those obtained by using conventional DTI as reported in past studies, the mean FA and ADC were in agreement $(0.587 \pm 0.023,0.928 \pm 0.111 \times$ $\left.10^{-3} \mathrm{~mm}^{2} \mathrm{~s}^{-1}\right),{ }^{7,18}$ whereas discrepancy existed in the mean $\lambda / /$ and $\lambda \perp(0.874 \pm$ $0.262 \times 10^{-3} \mathrm{~mm}^{2} \mathrm{~s}^{-1}, 2.088 \pm 0.136 \times$ $\left.10^{-3} \mathrm{~mm}^{2} \mathrm{~s}^{-1}\right) .^{7,19}$ As expected, $\lambda / /$ and $\lambda \perp$ are subject to larger variance subsequent to different scan protocols and hardware compared with ADC and FA. $^{7-9,18,19}$

FA measures the level of diffusion anisotropy and has been most widely used to assess the integrity of white matter tracts. ${ }^{7}$ Gradual loss of retinal ganglion cells in patients with RP takes place with the progressive death of photoreceptor cells, governed by the trans-synaptic neuronal degeneration mechanism, ${ }^{20-22}$ and may lead to axonal degeneration, axonal disruption or loss, and demyelination in the ONs of patients with RP. In chronic $\mathrm{ON}$ damage in RP, as the integrity of axon and myelin is compromised, $\mathrm{ON}$ tissue is no longer tightly packed and the widened interstitial space leads to decreased levels of diffusion anisotropy. This outcome explains the reduced $\mathrm{FA}$ for the $\mathrm{ON}$ in patients with RP compared with controls. These results were similar to those reported in glaucoma. ${ }^{8,23}$

VF loss in patients with RP has been attributed to the death of photoreceptors. ${ }^{4}$ With the death of photoreceptor cells and following loss of retinal ganglion cells, optic neuropathy of RP advances. VF defects may reflect ON damage in RP. The significant correlation of the VF defect with FA reduction in patients with RP suggests that the reduced anisotropy is of functional relevance and may reflect axonal disruption or loss and demyelination. A previous study by Khong et $\mathrm{al}^{24}$ showed that ADC was not as sensitive as FA for the assessment of neural tissue degeneration. This finding may explain ADC values for ONs in RP being higher than those in controls and not correlating with VF in our study. Another study ${ }^{23}$ in glaucoma also suggested that FA is more sensitive than ADC in assessing ON damage.

The $\lambda / /$ and $\lambda \perp$, as the biomarkers for the integrity of axons and myelin respectively, are commonly used to gather pathologic information of ON disease. ${ }^{13,25,26}$ In this study, both $\lambda / /$ and $\lambda \perp$ values for ONs in patients with RP were observed to be higher than those of controls; this finding likely indicates that axon and myelin injury might be linked. Because optic neuropathy in RP is 


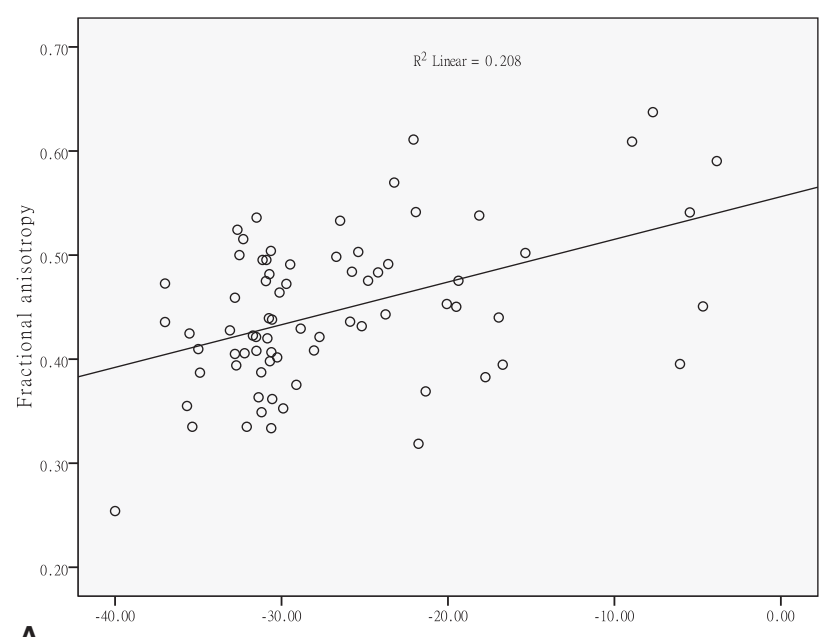

A

Mean deviation of visual field $(\mathrm{dB})$

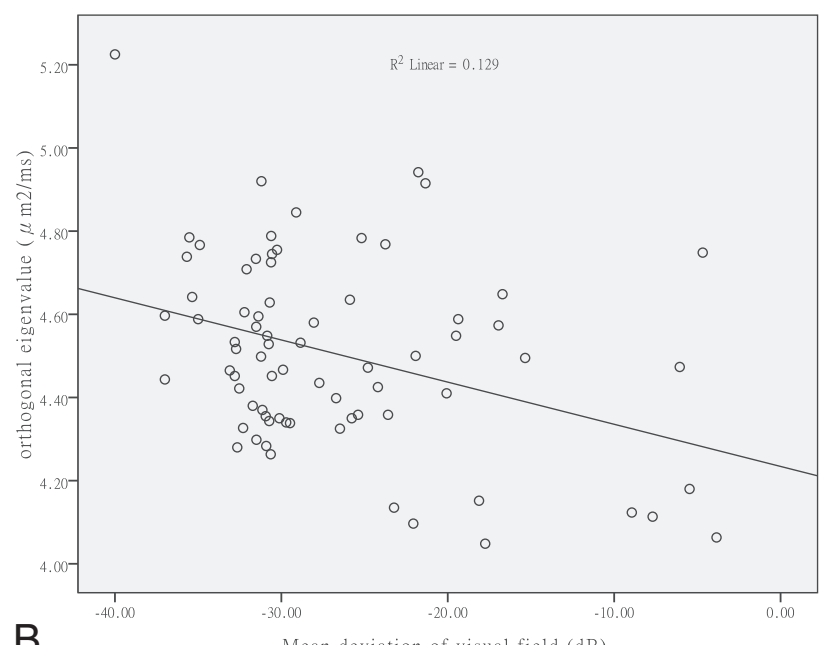

B

Mean deviation of visual field $(\mathrm{dB})$

FIG 4. Correlation of DTI data with visual function. Plot of FA and orthogonal eigenvalue versus mean deviation of the visual field, respectively, averaged across the pixels inside the ROls along the optic nerves of 38 patients with RP (76 affected eyes).

the result of gradual loss of retinal ganglion cells, axonal degeneration in the ON rather than demyelination might be the leading consequence of ON damage in patients with RP. However, our study showed that there was a significant correlation of $\lambda \perp$ with VF, but no correlation of $\lambda / /$ with VF in patients with RP. In general, in patients with RP, there is a delay between pathologic changes and clinically evident symptoms. ${ }^{11}$ Consequently, patients with RP diagnosed by typical symptoms in our study were likely manifesting a chronic stage of ON damage. A previous study $^{27}$ revealed that $\lambda / /$ becomes less informative as pathologic conditions of the human central nervous system progress from acute to chronic. Hence, changes of $\lambda / /$ in patients compared with controls were detected, but no correlation of $\lambda / /$ with visual function was seen, which was similar to the observation in our previous study in glaucoma. ${ }^{8}$

A previous study by Ohno et al ${ }^{1}$ using DTI showed correlation of FA in the optic radiation with VA but not with VF; in this study, results from rFOV-DTI showed correlation between FA for ONs and VF, but no statistically significant correlation was observed for any other parameters. This might be attributed to the patho- logic difference between the $\mathrm{ON}$ and optic radiation in patients with RP, and further studies are needed to better understand the trans-synaptic degeneration mechanism in RP.

There are several limitations to this study. First, there is a lack of correlation analysis between DTI measurements and the disease time course of RP, which could be an important factor in the damage of ONs. Only the age of onset of symptoms in RP was obtained, which is an imprecise measure of disease severity and gives little or no indication of the time point when photoreceptor degeneration actually began. ${ }^{11}$ Second, suppression of CSF was not performed, and the hyperintense signal may obscure signal changes of the $\mathrm{ON}$ and lead to potential underestimation of diffusion anisotropy measures such as FA. ${ }^{28}$ However, the consistency of measurements such as FA with those obtained by using acquisitions with both fat and CSF suppression ${ }^{19}$ supports the validity of the results. Third, the relatively long scan time ( $5 \mathrm{~min}$ utes 37 seconds) may still limit the clinical use of this technique, given the susceptibility to motion.

\section{CONCLUSIONS}

The present study demonstrated that rFOV-DTI measurements of the ON may reflect pathologic conditions in patients with RP and may serve as a biomarker of axonal and myelin damage in the optic neuropathy of RP. The use of rFOV-DTI in optic neuropathy of RP may allow early assessment of the status of the $\mathrm{ON}$ in this patient group. The alterations of ON reflected in rFOV-DTI measurements would also be valuable for assessing treatment trials in patients with RP.

\section{ACKNOWLEDGMENTS}

We are grateful to the staff at the Center of Translation Medicine, Zhengzhou University People's Hospital (Henan Provincial People's Hospital), for their assistance in this project.

Disclosures: Yanqiu Zhang, Ling Wang, Qin Tian, Dapeng Shi-RELATED: Grant: National Natural Science Foundation of China Grant (No. 81271534).* * Money paid to the institution.

\section{REFERENCES}

1. Ohno N, Murai H, Suzuki Y, et al. Alteration of the optic radiations using diffusion tensor MRI in patients with retinitis pigmentosa. Br J Ophthalmol 2015;99:1051-54 CrossRef Medline

2. Delyfer MN, Léveillard T, Mohand-Saïd S, et al. Inherited retinal degenerations: therapeutic prospects. Biol Cell 2004;96:261-69 CrossRef Medline

3. RetNet. The Retinal Information Network. https://sph.uth.edu/Retnet/. Accessed October 30, 2015

4. Walia S, Fishman GA, Edward DP, et al. Retinal nerve fiber layer defects in RP patients. Invest Ophthalmol Vis Sci 2007;48:4748-52 CrossRef Medline

5. Walia S, Fishman GA. Retinal nerve fiber layer analysis in RP patients using Fourier-domain OCT. Invest Ophthalmol Vis Sci 2008; 49:3525-28 CrossRef Medline

6. Eng JG, Agrawal RN, Tozer KR, et al. Morphometric analysis of optic nerves and retina from an end-stage retinitis pigmentosa patient with an implanted active epiretinal array. Invest Ophthalmol Vis Sci 2011;52:4610-16 CrossRef Medline

7. Wang MY, Qi PH, Shi DP. Diffusion tensor imaging of the optic nerve in subacute anterior ischemic optic neuropathy at 3T. AJNR Am J Neuroradiol 2011;32:1188-94 CrossRef Medline

8. Wang MY, Wu K, Xu JM, et al. Quantitative 3-T diffusion tensor imaging in detecting optic nerve degeneration in patients with 
glaucoma: association with retinal nerve fiber layer thickness and clinical severity. Neuroradiology 2013;55:493-98 CrossRef Medline

9. Wheeler-Kingshott CA, Trip SA, Symms MR, et al. In vivo diffusion tensor imaging of the human optic nerve: pilot study in normal controls. Magn Reson Med 2006;56:446-51 CrossRef Medline

10. Tang L, Wen Y, Zhou Z, et al. Reduced field-of-view DTI segmentation of cervical spine tissue. Magn Reson Imaging 2013;31:1507-14 CrossRef Medline

11. Hartong DT, Berson EL, Dryja TP. Retinitis pigmentosa. Lancet 2006;368:1795-809 CrossRef Medline

12. Murakami Y, Yoshida N, Ikeda Y, et al. Relationship between aqueous flare and visual function in retinitis pigmentosa. Am JOphthalmol 2015;159:958-63.el CrossRef Medline

13. Xu J, Sun SW, Naismith RT, et al. Assessing optic nerve pathology with diffusion MRI: from mouse to human. NMR Biomed 2008;21: 928-40 CrossRef Medline

14. Wheeler-Kingshott CA, Parker GJ, Symms MR, et al. ADC mapping of the human optic nerve: increased resolution, coverage, and reliability with CSF-suppressed ZOOM-EPI. Magn Reson Med 2002;47: 24-31 CrossRef Medline

15. Trip SA, Wheeler-Kingshott C, Jones SJ, et al. Optic nerve diffusion tensor imaging in optic neuritis. Neuroimage 2006;30:498-505 CrossRef Medline

16. Saritas EU, Cunningham CH, Lee JH, et al. DWI of the spinal cord with reduced FOV single-shot EPI. Magn Reson Med 2008;60: 468-73 CrossRef Medline

17. Attenberger UI, Rathmann N, Sertdemir M, et al. Small field-of-view single-shot EPI-DWI of the prostate: evaluation of spatially-tailored two-dimensional radiofrequency excitation pulses. $Z \mathrm{Med}$ Phys 2015 Aug 20. [Epub ahead of print] CrossRef Medline

18. Hickman SJ, Wheeler-Kingshott CA, Jones SJ, et al. Optic nerve diffusion measurement from diffusion-weighted imaging in optic neuritis. AJNR Am J Neuroradiol 2005;26:951-56 Medline
19. Techavipoo U, Okai AF, Lackey, et al. Toward a practical protocol for human optic nerve DTI with EPI geometric distortion correction. J Magn Reson Imaging 2009;30:699-707 CrossRef Medline

20. Gartner S, Henkind P. Pathology of retinitis pigmentosa. Ophthalmology 1982;89:1425-32 CrossRef Medline

21. Stone JL, Barlow WE, Humayun MS, et al. Morphometric analysis of macular photoreceptors and ganglion cells in retinas with retinitis pigmentosa. Arch Ophthalmol 1992;110:1634-39 CrossRef Medline

22. Humayun MS, Prince M, de Juan E Jr. Morphometric analysis of the extramacular retina from postmortem eyes with retinitis pigmentosa. Invest Ophthalmol Vis Sci 1999;40:143-48 Medline

23. Garaci FG, Bolacchi F, Cerulli A, et al. Optic nerve and optic radiation neurodegeneration in patients with glaucoma: in vivo analysis with 3-T diffusion-tensor MR imaging. Radiology 2009;252:496-501 CrossRef Medline

24. Khong PL, Zhou LJ, Ooi GC, et al. The evaluation of Wallerian degeneration in chronic paediatric middle cerebral artery infarction using diffusion tensor MR imaging. Cerebrovasc Dis 2004; 18:240 - 47 CrossRef Medline

25. Song SK, Sun SW, Ju WK, et al. Diffusion tensor imaging detects and differentiates axon and myelin degeneration in mouse optic nerve after retinal ischemia. Neuroimage 2003;20:1714-22 CrossRef Medline

26. Michielse S, Coupland N, Camicioli R, et al. Selective effects of aging on brain white matter microstructure: a diffusion tensor imaging tractography study. Neuroimage 2010;52:1190-201 CrossRef Medline

27. Naismith RT, Xu J, Tutlam NT, et al. Disability in optic neuritis correlates with diffusion tensor-derived directional diffusivities. Neurology 2009;72:589-94 CrossRef Medline

28. Bhagat YA, Beaulieu C. Diffusion anisotropy in subcortical white matter and cortical gray matter: changes with aging and the role of CSF-suppression. J Magn Reson Imaging 2004;20:216-27 CrossRef Medline 